\title{
Human endogenous retrovirus-H insertion screening
}

\author{
MEHRAB GULIYEV, SIBEL YILMAZ, KANIYE SAHIN, SEVGI MARAKLI and NERMIN GOZUKIRMIZI
}

Department of Molecular Biology and Genetics, Faculty of Science, Istanbul University, Vezneciler, Istanbul 34134, Turkey

Received October 22, 2012; Accepted January 22, 2013

DOI: $10.3892 / \mathrm{mmr} .2013 .1295$

\begin{abstract}
Endogenous retroviruses (ERVs) and ERV-like sequences comprise $8 \%$ of the human genome. We aimed to analyze genome integration polymorphisms of human endogenous retrovirus (HERV)-H by the inter-retrotransposon amplified polymorphism (IRAP) technique using the sequences of LTR7A (450 bp), LTR7B (445 bp) and LTR7C (471 bp). Blood samples from 20 individuals (10 females and 10 males) of diverse ethnic origins were used for the determination of integration variations at the genomic level. Isolated genomic DNA was screened using 3 pairs of primers corresponding to LTR regions of the HERV-H gene. We observed insertion polymorphism patterns between $0-87 \%$ in all subjects. The findings obtained contribute to our understanding of the effects of HERV-H on variations within the human genome.
\end{abstract}

\section{Introduction}

Retroviruses, found in all mammals and in a wide range of other vertebrates, provide unique opportunities to study the biology and evolution of virus-host relationships. Occasionally, infection of a germline cell by a retrovirus may lead to an integrated provirus that is passed to the offspring and inherited in a Mendelian fashion; known as an endogenous retrovirus (ERV). Human endogenous retroviruses (HERVs) constitute $\sim 8 \%$ of the human genome and are distributed in $\sim 700,000$ different loci (1). Expression of HERVs has been associated with several positive physiological functions as well as certain diseases, although their role as an etiological agent, a possible contributing factor or a disease marker remains to be established (2).

The proviral structure of HERV elements mainly consists of 5'LTR-gag-pro-pol-env-3'LTR, in which the 4 genes (gag, group-specific antigen; pro, protease; pol, polymerase; and $e n v$, envelope) encode structural/functional proteins

Correspondence to: Professor Nermin Gozukirmizi, Department of Molecular Biology and Genetics, Faculty of Science, Istanbul University, Vezneciler Street, Vezneciler, Istanbul 34134, Turkey E-mail: nermin@istanbul.edu.tr

Key words: inter-retrotransposon amplified polymorphism, human endogenous retrovirus- $\mathrm{H}$, genetic diversity, population screening essential to a replication-competent retrovirus (3). The long terminal repeats (LTRs) at both ends differentiate HERVs from other retrotransposons, such as long interspersed nuclear elements (LINEs). The majority of the remnants of HERVs are simply isolated LTR copies, with the internal sequence having been lost during integration or via homologous recombination. HERV families are defined by different criteria, such as homogeneity to their exogenous counterparts, sequence similarity of the pol genes and the primer binding site (PBS) immediately downstream of the 5'LTR. H family HERV (HERV-H) contain a PBS with a sequence similar to human tRNA ${ }^{\text {His }}$ (4).

HERV-H is one of the most abundant endogenous retroviral families in the human genome, consisting of full-length elements $(\sim 100$ copies, of which only 18 are relatively complete) (3), elements deleted in pol and env [800-900 copies, of which only 3 have been identified to contain intact $e n v$ open reading frames (ORFs)] (5), and solitary LTRs $(\sim 1,000$ copies) (6). HERV-H was inserted into primate genomes prior to the divergence of New and Old World monkeys, and the elements lacking in pol and $e n v$ were integrated with the Old World monkey lineage $(5,7,8)$. HERV-H env fragments are found on human chromosomes 1, 2, 3, 4, 5, 6, 7, 9, 10, 11, 12, 14, $15,16,17,18,19,20, \mathrm{X}$ and Y. These new HERV-H env fragments showed 82-99\% sequence similarity to that of HERV-H. The HERV-H members evolved by intra-chromosomal spread during hominid radiation (9).

Using the inter-retrotransposon amplified polymorphism (IRAP) technique, which is one of the retrotransposon-based markers, regions flanked by two LTR-retrotransposons or solo LTRs are amplified (10). In this method, polymorphisms are detected by the presence or absence of the PCR product, where the lack of amplification indicates the absence of a retrotransposon at that particular region (11). IRAP was used to investigate genetic relationships between different and related species (12), for gene mapping (13) and for the characterization of the somaclonal variants in plants (14-16).

ERVs differ between host species, most likely in correlation with differences in expression conditions, and in the evolutionary and environmental history of each host (17). There are very limited data on environmentally and developmentally (ED) regulated genomic variation. Further research is required to understand the cytogenetic, DNA-structural and gene regulation basis of ED-genomic variation, and to study the importance of ED-genomic variation for population genetics, adaptation, domestication, evolution, medical science, plant science and agriculture (18). 
Table I. Evaluation forms of subjects.

\begin{tabular}{|c|c|c|c|c|c|}
\hline \multirow[b]{2}{*}{ Subject } & \multirow[b]{2}{*}{ Gender } & \multirow[b]{2}{*}{ Age (years) } & \multirow[b]{2}{*}{ Ethnic group } & \multicolumn{2}{|c|}{ Exposure to } \\
\hline & & & & Radiation & Infectious disease \\
\hline 1 & $\mathrm{M}$ & 28 & Asia (Azerbaijan) & $\mathrm{x}$ & \\
\hline 2 & $\mathrm{M}$ & 21 & Asia (Azerbaijan) & $\mathrm{x}$ & \\
\hline 3 & M & 19 & Asia (Azerbaijan) & $\mathrm{x}$ & $\mathrm{x}$ \\
\hline 4 & $\mathrm{~F}$ & 24 & Asia (Azerbaijan) & & $\mathrm{x}$ \\
\hline 5 & M & 26 & Asia (Azerbaijan) & & \\
\hline 6 & $\mathrm{~F}$ & 23 & Asia (Azerbaijan) & & \\
\hline 7 & $\mathrm{M}$ & 28 & Asia (Azerbaijan) & $\mathrm{x}$ & \\
\hline 8 & $\mathrm{M}$ & 28 & Asia (Iran - Azerbaijan) & $\mathrm{x}$ & \\
\hline 9 & $\mathrm{~F}$ & 23 & Asia (Turkey) & & \\
\hline 10 & $\mathrm{~F}$ & 25 & Asia (China-Eastern Turkmenistan) & $\mathrm{x}$ & $\mathrm{x}$ \\
\hline 11 & $\mathrm{~F}$ & 23 & Asia (Turkey) & & \\
\hline 12 & M & 22 & Asia (Turkey) & & $\mathrm{x}$ \\
\hline 13 & $\mathrm{~F}$ & 22 & Asia (Turkey) & $\mathrm{x}$ & \\
\hline 14 & $\mathrm{M}$ & 22 & Asia (Turkey) & & \\
\hline 15 & $\mathrm{~F}$ & 22 & Asia (Turkey) & $\mathrm{x}$ & \\
\hline 16 & M & 23 & Asia (Turkey) & & $\mathrm{x}$ \\
\hline 17 & $\mathrm{~F}$ & 20 & Asia (Turkey) & & \\
\hline 18 & F & 20 & Asia (Indonesia) & $\mathrm{x}$ & \\
\hline 19 & $\mathrm{~F}$ & 22 & Africa (Somali) & & \\
\hline 20 & $\mathrm{M}$ & 24 & Asia (Turkey) & $\mathrm{x}$ & \\
\hline
\end{tabular}

In recent years, there have been a number of studies on the different expression profiles of HERV-H in various clinical situations $(3,19,20)$. However, there are no data on HERV-H provirus integration variations between healthy individuals. In this study, we report the HERV-H integration polymorphisms between healthy individuals.

\section{Materials and methods}

Subjects. This study included 20 individuals (10 males and 10 females; age, 18-30 years) with different ethnic origins (Turkey, Azerbaijan, Indonesia, China and Somalia; Table I). The study design was approved as a Master's Thesis (Project no. T-17640) by the Istanbul University, Istanbul, Turkey. The blood samples were collected by the Istanbul University Medico-Social Center, Istanbul, Turkey. Blood samples were obtained by the Istanbul University Medico-Social Center, Turkey, with the written consent of the healthy individuals.

Genomic DNA isolation. Genomic DNA was extracted from blood samples using a High Pure PCR Template Preparation kit (Roche Diagnostics, Mannheim, Germany). The DNA concentrations were measured using a spectrophotometer (Nanodrop2000) and genomic DNA samples were separated on an EtBr-stained, 1\% agarose gel to estimate the quality of the DNA.

IRAP analysis. HERV-H integration polymorphisms were investigated using the IRAP technique. LTR primers were used for IRAP analysis. The primers shown in Table II were designed using the online primer design site of Integrated DNA Technologies (http://eu.idtdna.com/PrimerQuest/Home/ Index). A pair of primers was designed for each LTR7A, LTR7B and LTR7C region. HERV-H LTR sequences were obtained from a relevant database (www.girinst.org, AC D11078). PCR was performed using a thermal cycler (Techne, TC3000) in a total volume of $20 \mu \mathrm{l}$, containing $75 \mathrm{ng}$ of template DNA, $10 \mu \mathrm{M}$ of forward and reverse primers and SapphireAmp Master Mix (2X). PCR conditions were as follows: initial denaturation at $95^{\circ} \mathrm{C}(3 \mathrm{~min})$ followed by 30 cycles of denaturation at $94^{\circ} \mathrm{C}(30 \mathrm{sec})$, annealing at $65^{\circ} \mathrm{C}$ for LTR7A, $68.2^{\circ} \mathrm{C}$ for LTR7B and $67^{\circ} \mathrm{C}$ for LTR7C $(30 \mathrm{sec})$ and extension at $72^{\circ} \mathrm{C}$ ( $3 \mathrm{~min}$ ). The reaction was completed by an additional extension at $72^{\circ} \mathrm{C}$ for $5 \mathrm{~min}$. A total of $20 \mu \mathrm{l}$ of IRAP-PCR products were mixed with $4 \mu \mathrm{l} 6 \mathrm{X}$ loading buffer $(10 \mathrm{mM}$ Tris- $\mathrm{HCl}$, 60 mM EDTA, pH 8.0,0.3\% bromophenol blue, 60\% glycerol) and resolved by $2 \%$ agarose gel electrophoresis at $180 \mathrm{~V}$ for $2 \mathrm{~h}$ in 1X TAE buffer (90 mM Tris, $90 \mathrm{mM}$ boric acid and $2 \mathrm{mM}$ EDTA, pH 8.0). A molecular weight marker (GeneRuler ${ }^{\mathrm{TM}}$ DNA Ladder Mix, SM0331, Fermentas, Waltham, MA, USA) was also loaded to determine the size of the amplicons. After running, the gels were photographed on a UV transilluminator and analyzed visually using Kodak Molecular Imaging Software (1994-2007 Carestream Health, Inc., Rochester, NY, USA).

Statistical analysis. Polymorphism rates were calculated using Jaccard similarity coefficient and the scores were evaluated statistically by ANOVA test. $\mathrm{P}<0.05$ was considered to indicate a statistically significant difference. 
Table II. Primers used for IRAP analyses for HERV-H integration polymorphism study.

\begin{tabular}{lccc}
\hline Primers & $\mathrm{G}+\mathrm{C}$ ratio $(\%)$ & $\mathrm{Tm}\left({ }^{\circ} \mathrm{C}\right)$ & Sequence $\left(5^{\prime}-3^{\prime}\right)$ \\
\hline LTR7A & & & \\
Forward & 50 & 72 & TGTTTGGTGGTCTCTTCACACGGA \\
Reverse & 50 & 72 & ATGGTATGGCTTAGCTTGGGCTCA \\
LTR7B & & 72 & TGTTTGGTGGTCTCTTCACACGGA \\
Forward & 50 & 72 & ATGATGGCTTAGCTTGGGCTCAGA \\
Reverse & 50 & & \\
LTR7C & & 72 & TTGCACCCAAGTGAATAAACGGCC \\
Forward & 50 & 72 & TTACAATGGCTGAGCTTCGGCTCA \\
Reverse & 50 &
\end{tabular}

IRAP, inter-retrotransposon amplified polymorphism; HERV-H, human endogenous retrovirus-H; Tm, melting temperature.

$\begin{array}{lllllllllllllllllllllll}\text { M } & 01 & 02 & 03 & 04 & 05 & 06 & 07 & 08 & 09 & 10 & 11 & 12 & 13 & 14 & 15 & 16 & 17 & 18 & 19 & 20 & \text { M NC }\end{array}$

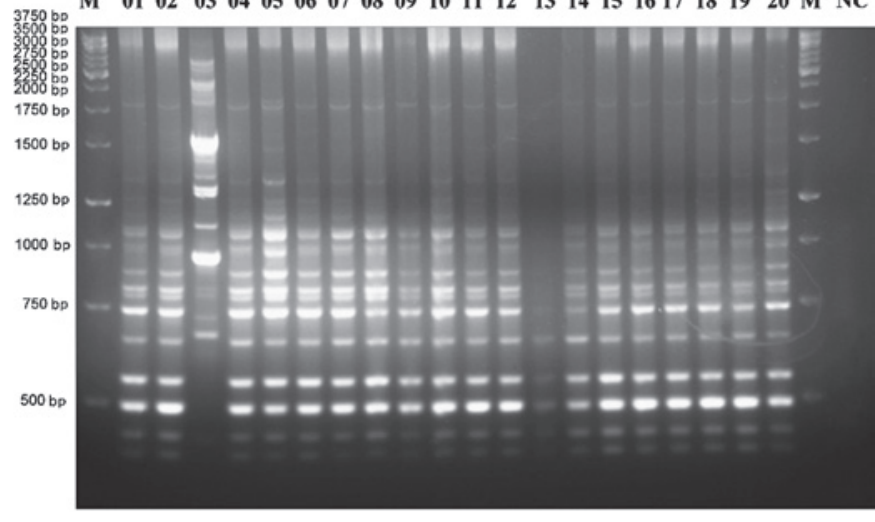

M,marker; 01-20, sample; NC, negative control

Figure 1. Inter-retrotransposon amplified polymorphism (IRAP)-PCR analyses using human endogenous retrovirus (HERV)-H LTR7A primers.

\section{Results}

IRAP-PCR analyses using HERV-H LTR7A primers with 20 human DNA samples are shown in Fig. 1. Kodak Molecular Imaging Software analyses are presented in Fig. 2. As can be observed in Fig. 2, polymorphisms between individuals were between $0-86 \%$. Under the same experimental conditions with HERV-H LTR7B primers, we also observed polymorphisms (Fig. 3). Using Kodak Molecular Imaging Software, the polymorphism rate between the individuals was calculated to be between $0-87 \%$ with HERV-H LTR7B (Fig. 2). These polymorphism rates were used to calculate the $\mathrm{p}$ value by ANOVA test. The polymorphism rates between individuals were found to be significant $(\mathrm{P} \leq 0.05)$. However, we were not able to detect any amplification products using HERV-H LTR7C primers.

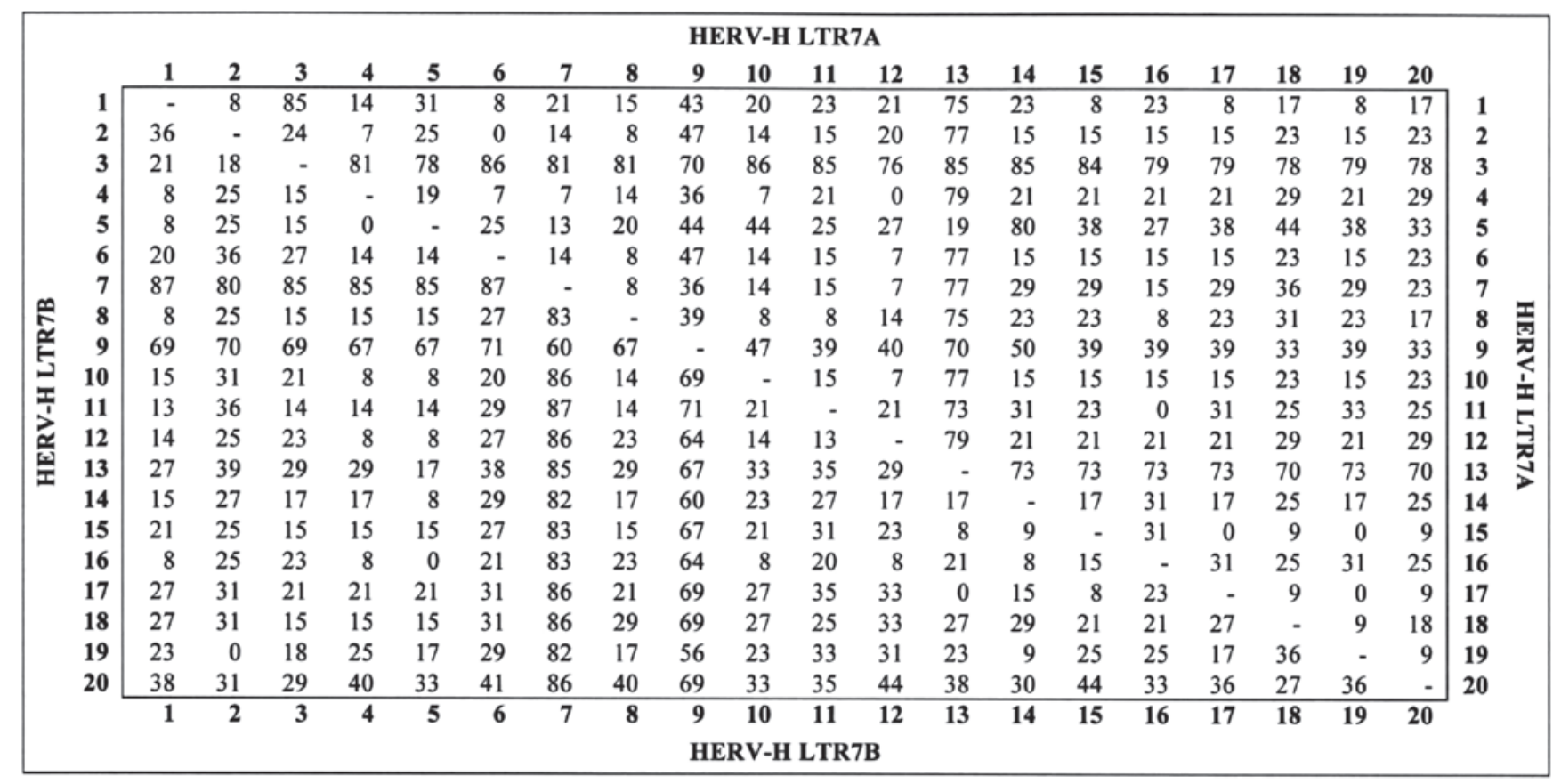


M 0102030405060708091011121314151617181920 M NC

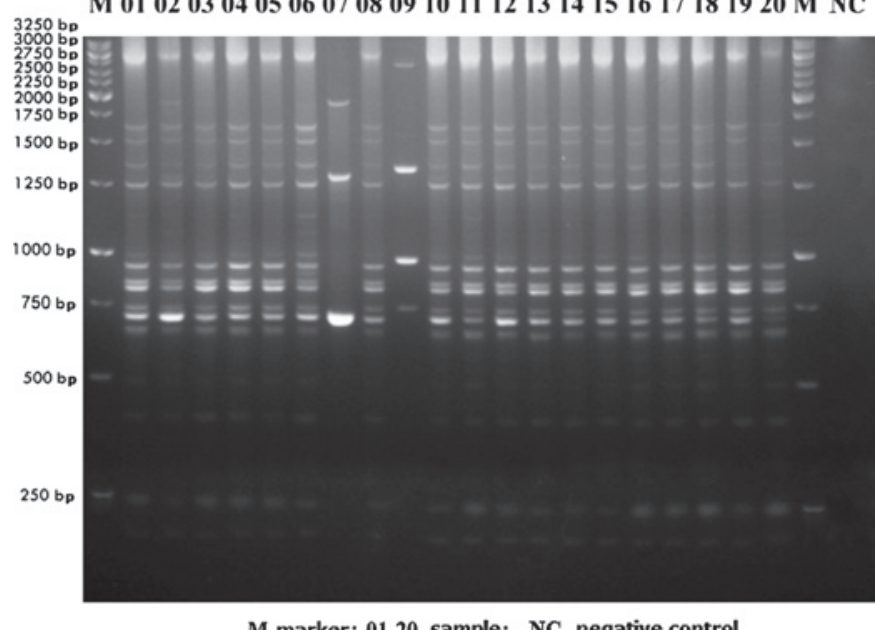

Figure 3. Inter-retrotransposon amplified polymorphism (IRAP)-PCR analyses using human endogenous retrovirus (HERV)-H LTR7B primers.

No significant data were obtained by comparison of the ethnic groups. In the Azerbaijani group, variations were calculated to be between $2.65-9.73 \%$ in terms of HERV-H LTR7A and $1.40-5.63 \%$ in terms of HERV-H LTR7B.

\section{Discussion}

In the present study, LTR7A (450 bp), LTR7B (445 bp) and LTR7C (471 bp) regions of HERV-H were analyzed by IRAP-PCR and the integration patterns between the individuals were compared. Blood samples from 20 individuals of various ethnic origins were used for determination of the integration variations at the genomic level. Isolated genomic DNA was screened using 3 pairs of primers covering LTR regions of the HERV-H gene. The IRAP technique was performed for polymorphism analyses. We observed integration polymorphism patterns in all the tested individuals. To date, there has been no report on HERV-H integration polymorphisms between individuals; however, there are limited reports on integration polymorphisms of HERV-K families (21-23). Recently, database documentation relating to Alu retrotransposon insertion polymorphisms (RIPs) was presented (24).

Insertion polymorphism of retroelements (REs) has also attracted considerable attention due to certain advantageous features that make REs useful tools for human population genetic studies (25-27). These include known ancestral state (absence of the RE), stability of insertion (there are no mechanisms for removing inserted repeats) and relatively easy detection. Moreover, an event of two independent integrations into one and the same locus is very improbable, making this type of polymorphism essentially homoplasy-free $(28,29)$. Previously, a number of cases of RIPs have been studied for evolutionarily young groups of short interspersed elements (SINEs) and long interspersed elements (LINEs) $(30,31)$. Although insertion polymorphisms of HERVs appear to be a rare event, in our study we observed a high number of insertion polymorphisms between individuals in terms of HERV-H insertion. We were not able to observe significant differences between ethnic groups, since we observed different degrees of polymorphisms between all the test subjects.
The HERV-H family is the largest group among the HERVs $(19,32)$ with 100 copies of full-length HERV-H elements, 800-900 copies of HERV-H lacking pol and env genes and solitary LTRs (up to 1000 copies) in the human genome $(6,19)$. HERV-H elements integrated into the primate genome 40 million years ago and their numbers have been increasing over the past 30-35 million years $(8,19,32)$. Our results showed that HERV-H is still active in the human genome. These data are also important from an evolutionary point of view.

HERV-H contains regions of the gene caused by mutations known to be due to integration into the human genome. An important gene mutation of retroviruses in the env ORF was detected (33). The total size of HERV-H is $9 \mathrm{~kb}$ in the human genome and HERV-H has ORFs for gag, pro, pol and env genes. LTR sequences have variants of $1.1 \%$ due to mutations in the $e n v$ gene. Research on the $e n v$ gene has demonstrated new alternative reading frames $(8,22)$ and $e n v$ expression was observed in normal tissues and colon tumors.

To date, HERV-H research has been focused in particular on investigating the relationship between neurological diseases, cancer and gene expression $(19,20)$. In our study we were able to observe, for the first time, that major genomic variations between individuals derive from the integration of various elements. These polymorphic HERVs may be associated with diseases. A gene that has an RIP may have potential alternative transcripts, a promoter activity of alternative splice sites, or create a poly-A signal (35).

IRAP is a valuable technique for searching the retrotransposon movements in the plant kingdom $(11,15)$. We also demonstrated that this valuable technique can be successfully applied to human research. Although the experiment was conducted with a limited number of subjects, we were able to observe polymorphisms and variants of inter-retrotransposon regions. Our data may contribute to the understanding of the variation of HERV-H in human populations. This study provides new research areas on possible integration sites, and the existence of infectious HERV-H variants may also be considered.

\section{Acknowledgements}

This study was supported by the Istanbul University Research Foundation Project (no. T-17640).

\section{References}

1. Lander ES, Linton LM, Birren B, et al: Initial sequencing and analysis of the human genome. Nature 409: 860-921, 2001.

2. Jern P and Coffin JM: Effects of retroviruses on host genome function. Annu Rev Genet 42: 709-732, 2008.

3. Jern P, Sperber GO, Ahlsén G and Blomberg J: Sequence variability, gene structure, and expression of full-length human endogenous retrovirus H. J Virol 79: 6325-6337, 2005.

4. Tristem M: Identification and characterization of novel human endogenous retrovirus families by phylogenetic screening of the human genome mapping project database. J Virol 74: 3715-3730, 2000.

5. de ParsevalN,CasellaJ,Gressin LandHeidmannT: Characterization of the three HERV-H proviruses with an open envelope reading frame encompassing the immunosuppressive domain and evolutionary history in primates. Virology 279: 558-569, 2001.

6. Hirose Y, Takamatsu M and Harada F: Presence of env genes in members of the RTVL-H family of human endogenous retrovirus-like elements. Virology 192: 52-61, 1993. 
7. Goodchild N, Wilkinson DA and Mager D: Recent evolutionary expansion of a subfamily of RTVL-H human endogenous retrovirus-like elements. Virology 196: 778-788, 1993.

8. Anderssen S, Sjøttem E, Svineng G and Johansen T: Comparative analyses of LTRs of the ERV-H family of primate-specific retrovirus-like elements isolated from marmoset, African green monkey, and man. Virology 234: 14-30, 1997.

9. Yi JM and Kim HS: Evolutionary implications of human endogenous retrovirus HERV-H family. J Hum Genet 49: 215-219, 2004

10. Schulman AH and Kalendar R: A movable feast: diverse retrotransposons and their contribution to barley genome dynamics. Cytogenet Genome Res 110: 598-605, 2005.

11. Kalendar R, Grob T, Regina M, Suoniemi A and Schulman A: IRAP and REMAP: two new retrotransposon-based DNA fingerprinting techniques. Theor Appl Genet 98: 704-711, 1999.

12. Guo D, Zhang H and Luo Z: Genetic relationships of Diospyros kaki Thunb. and related species revealed by IRAP and REMAP analysis. Plant Sci 170: 528-533, 2006.

13. Manninen O, Kalendar R, Robinson $\mathrm{J}$ and Schulman AH: Application of $B A R E-1$ retrotransposon markers to the mapping of a major resistance gene for net blotch in barley. Mol Gen Genet 264: 325-334, 2000.

14. Muhammad AJ and Othman RY: Characterization of Fusarium wilt-resistant and Fusarium wilt-susceptible somaclones of banana cultivar rastali (Musa AAB) by random amplified polymorphic DNA and retrotransposon markers. Plant Mol Biol Rep 23: 241-249, 2005.

15. Evrensel C, Y1lmaz S, Temel A and Gozukirmizi N: Variations in $B A R E-1$ insertion patterns in barley callus cultures. Genet Mol Res 10: 980-987, 2011.

16. Bayram E, Yilmaz S, Hamat-Mecbur H, Kartal-Alacam G and Gozukirmizi N: Nikita retrotransposon movements in callus cultures of barley (Hordeum vulgare L.). POJ 5: 211-215, 2012.

17. Grandbastien MA, Audeon C, Bonnivard E, et al: Stress activation and genomic impact of Tnt1 retrotransposons in Solanaceae. Cytogenet Genome Res 110: 229-241, 2005.

18. Li XQ: Developmental and environmental variation in genomes Heredity (Edinb) 102: 323-329, 2009.

19. Yi JM, Kim HM and Kim HS: Human endogenous retrovirus HERV-H family in human tissues and cancer cells: expression, identification and phylogeny. Cancer Lett 231: 228-239, 2006.

20. Laska MJ, Brudek T, Nissen KK, Christensen T, MøllerLarsen A, Petersen T and Nexø BA: Expression of HERV-Fc1, a human endogenous retrovirus, is increased in patients with active multiple sclerosis. J Virol 86: 3713-3722, 2012

21. Mamedov I, Lebedev Y, Hunsmann G, Khusnutdinova E and Sverdlov E: A rare event of insertion polymorphism of a HERV-K LTR in the human genome. Genomics 84: 596-599, 2004
22. Belshaw R, Dawson AL, Woolven-Allen J, Redding J, Burt A and Tristem M: Genomewide screening reveals high levels of insertional polymorphism in the human endogenous retrovirus family HERV-K (HML2): implications for present-day activity. J Virol 79: 12507-12514, 2005

23. Moyes D, Griffiths DJ and Venables PJ: Insertional polymorphisms: a new lease of life for endogenous retroviruses in human disease. Trends Genet 23: 326-333, 2007.

24. Liang P and Tang W: Database documentation of retrotransposon insertion polymorphisms. Front Biosci (Elite Ed) 4: 1542-1555, 2012.

25. Batzer MA and Deininger PL: Alu repeats and human genomic diversity. Nat Rev Genet 3: 370-379, 2002

26. Watkins WS, Rogers AR, Ostler C, et al: Genetic variation among world populations: inference from 100 Alu insertion polymorphisms. Genome Res 13: 1607-1618, 2003.

27. Bamshad MJ, Wooding S, Watkins WS, Ostler CT, Batzer MA and Jorde LB: Human population genetic structure and inference of group membership. Am J Hum Genet 72: 578-589, 2003.

28. Roy-Engel AM, Carroll ML, El-Sawy M, Salem AH, Garber RK Nguyen SV, Deininger PL and Batzer MA: Non-traditional Alu evolution and primate genomic diversity. J Mol Biol 316: 1033-1040, 2002

29. Vincent BJ, Myers JS, Ho HJ, Kilroy GE, Walker JA, Watkins WS, Jorde LB and Batzer MA: Following the LINEs: an analysis of primate genomic variation at human-specific LINE-1 insertion sites. Mol Biol Evol 20: 1338-1348, 2003.

30. Carroll ML, Roy-Engel AM, Nguyen SV, et al: Large-scale analysis of the Alu Ya5 and Yb8 subfamilies and their contribution to human genomic diversity. J Mol Biol 311: 17-40, 2001.

31. Myers JS, Vincent BJ, Udall H, et al: A comprehensive analysis of recently integrated human Ta L1 elements. Am J Hum Genet 71: 312-326, 2002

32. Mager DL and Freeman JD: HERV-H endogenous retroviruses: prescence in the New World branch but amplification in the Old World primate lineage. Virology 213: 395-404, 1995.

33. Lindeskog M, Mager DL and Blomberg J: Isolation of a human endogenous retroviral HERV-H element with an open env reading frame. Virology 258: 441-450, 1999.

34. Huang AY, Gulden PH, Woods AS, et al: The immunodominant major histocompatibility complex class I-restricted antigen of a murine colon tumor derives from an endogenous retroviral gene product. Proc Natl Acad Sci USA 93: 9730-9735, 1996.

35. Sin HS, Huh JH, Kim WY, et al: Long terminal repeats of human endogenous retrovirus $\mathrm{H}$ family provide alternative poly-adenylation signals to NADSYN1 gene. Korean J Genet 29: 395-401, 2007. 